\title{
Der Rauch hat sich verzogen
}

... und wir staunen: Zigaretten werden spürbar teurer (woran wir uns schon gewöhnt haben), und ein Präventionsfonds, jahrelang schon im Gespräch, hat eine sichere Mehrheit gefunden im Nationalrat. Mit rauchfreier Klarheit - so der Kommentar der NZZ - hat nun diese Mehrheit die Argumente der Gesundheitsseite akzeptiert.

Es waren immer schon Ärzte, die neues im Tabakbereich zur Sprache brachten, vom TerryReport bis hin zum Antrag für den schweizerischen Präventionsfonds. Rauchen anzusprechen, ist heute Inbegriff ärztlichen Handelns.

Immer gründlich, manchmal frech, listig oft, haben sie Ideen und Konzepte vorgestellt und mit Ausdauer weiterverfolgt.

Lange zögerte die Politik hinzuhören. Zu präsent waren die Argumente um Toleranz und Freiheitlichkeit. Es sind dies die Argumente der Tabakindustrie, vielmehr als die der Rauchenden selber. Unterstützt von den riesigen Budgets hinter jenen Argumenten, haben die Zwillingsinitiativen und andere, eigentlich einsichtige Vorstösse keine Mehrheit gefunden, trotz der nichtrauchenden Mehrheit der Bevölkerung.

Jetzt haben Gesundheitsargumente gestochen: Ernsthaftigkeit dem Rauchenden und seiner Selbstgefährdung gegenüber ist geboten. Die vielen Widersprüche um die Zigaretten müssen reduziert werden, zum Schutz der Rauchenden. Schutz vor Passivrauchen und der Schutz von Kindern oder Lungenkranken kann ernsthaft nicht länger unbeachtet bleiben.
Die Schräglage in der Tabakpolitik besteht ja insbesondere im Weiterbestehen der Subventionen für den Tabakanbau, auf europäischer Ebene so stossend wie in den schweizerischen Dimensionen - bei gleichzeitig bekanntermassen horrenden Kosten des Rauchens im Gesundheitsbereich.

Deswegen wird der Entscheid des Nationalrates auch die beantragten Mittel für die Gesundheitsseite bewirken, ob nun elegant über das ordentliche Budget oder bauernschlau über den Fonds, der den SOTA-Fonds für die Tabakbauern ausgleicht. Es ist zu stossend, woran wir uns so lang gewöhnt haben.

Die Ärzteschaft der Schweiz engagiert sich auch erneut in der Tabakprävention: Im September 2002 hat das Fortbildungsprogramm «Ärztliche Rauch-Entwöhnung» der Lungenliga Schweiz, ein Projekt im Rahmen der nationalen Kampagne «Rauchen schadet», begonnen: Mit vorerst 20 Ärztinnen und Ärzten aus der ganzen Schweiz wird im Multiplikatorenprinzip in Qualitätszirkeln und Fortbildungskreisen zur kurzen und effizienten Raucherberatung angeleitet.

Anne-Katharina Burkhalter, Leitung Tabakprävention, Projekt «Ärztliche Rauch-Entwöhnung», Lungenliga Schweiz 